\title{
Combination of Prehospital NT-proBNP with qSOFA and NEWS to Predict Sepsis and Sepsis-Related Mortality
}

\author{
Francisco Martín-Rodríguez $\mathbb{D}^{1,2}$ Laura Melero-Guijarro, ${ }^{3}$ Guillermo J. Ortega, ${ }^{4}$ \\ Ancor Sanz-García $\mathbb{D}^{\mathrm{D}}{ }^{4}$ Teresa de la Torre de Dios, ${ }^{5}$ Jesús Álvarez Manzanares $\mathbb{D}^{6}{ }^{6}$ \\ José L. Martín-Conty $\mathbb{D}^{\mathbb{7}}{ }^{7}$ Miguel A. Castro Villamor $\mathbb{D}^{\mathbb{D}}{ }^{2}$ Juan F. Delgado Benito $\mathbb{D}^{\mathbb{D}}{ }^{1}$ \\ and Raúl López-Izquierdo (i) \\ ${ }^{1}$ Unidad Móvil de Emergencias, Gerencia de Emergencias Sanitarias, Gerencia Regional de Salud de Castilla y León (SACYL), Spain \\ ${ }^{2}$ Centro de Simulación Clínica Avanzada, Departamento de Medicina, Dermatología y Toxicología, \\ Universidad de Valladolid, Spain \\ ${ }^{3}$ Servicio de Urgencias, Complejo Asistencial Universitario de Palencia, \\ Gerencia Regional de Salud de Castilla y León (SACYL), Spain \\ ${ }^{4}$ Unidad de Análisis de Datos (UAD) del Instituto de Investigación Sanitaria del Hospital de la Princesa (IIS-IP), Madrid, Spain \\ ${ }^{5}$ Gerencia de Atención Primaria de Salamanca, Gerencia Regional de Salud de Castilla y León (SACYL), Spain \\ ${ }^{6}$ Servicio de Urgencias, Hospital Universitario Rio Hortega de Valladolid, \\ Gerencia Regional de Salud de Castilla y León (SACYL), Spain \\ ${ }^{7}$ Faculty of Health Sciences, Universidad de Castilla la Mancha, Talavera de la Reina, Spain
}

Correspondence should be addressed to Francisco Martín-Rodríguez; fmartin@saludcastillayleon.es and Ancor Sanz-García; ancor.sanz@gmail.com

Received 7 January 2022; Revised 8 February 2022; Accepted 9 February 2022; Published 23 February 2022

Academic Editor: Silvia Spoto

Copyright (c) 2022 Francisco Martín-Rodríguez et al. This is an open access article distributed under the Creative Commons Attribution License, which permits unrestricted use, distribution, and reproduction in any medium, provided the original work is properly cited.

Background. The aim of this study was to assess the role of prehospital point-of-care $N$-terminal probrain natriuretic peptide to predict sepsis, septic shock, or in-hospital sepsis-related mortality. Methods. A prospective, emergency medical service-delivered, prognostic, cohort study of adults evacuated by ambulance and admitted to emergency department between January 2020 and May 2021. The discriminative power of the predictive variable was assessed through a prediction model trained using the derivation cohort and evaluated by the area under the curve of the receiver operating characteristic on the validation cohort. Results. A total of 1,360 patients were enrolled with medical disease in the study. The occurrence of sepsis, septic shock, and in-hospital sepsis-related mortality was 6.4\% (67 cases), 4.2\% (44 cases), and 6.1\% (64 cases). Prehospital National Early Warning Score 2 had superior predictive validity than quick Sequential Organ Failure Assessment and $\mathrm{N}$-terminal probrain natriuretic peptide for detecting sepsis and septic shock, but $N$-terminal probrain natriuretic peptide outperformed both scores in in-hospital sepsis-related mortality estimation. Application of $N$-terminal probrain natriuretic peptide to subgroups of the other two scores improved the identification of sepsis, septic shock, and sepsis-related mortality in the group of patients with low-risk scoring. Conclusions. The incorporation of $\mathrm{N}$-terminal probrain natriuretic peptide in prehospital care combined with already existing scores could improve the identification of sepsis, septic shock, and sepsis-related mortality.

\section{Background}

Detection and quick response by the emergency medical services (EMS) of time-dependent illness can make a big differ- ence in the patient's condition [1]. Certain medical emergencies present a marked clinical manifestation, e.g., trauma, myocardial infarction, or stroke; all of them have specific codes and action guidelines already implemented 
to help in the management of these pathophysiological conditions [2] [3] [4]. However, other syndromic conditions such as sepsis present a more diffuse manifestation that may sometimes be unnoticed in its initial stages [5] [6].

The Third International Consensus Definitions for Sepsis and Septic Shock (Sepsis-3) [7] [8] and the International Guidelines for Management of Sepsis and Septic Shock 2021 "Surviving Sepsis Campaign" [9] [10] established the identification strategies and sequential lines of care to be followed in such cases. Hospital-based strategies have been developed for the early screening of sepsis, both in the intensive care units (ICU), such as the Sequential Organ Failure Assessment (SOFA) scores [11], and out of the ICU, such as the quick Sequential Organ Failure Assessment (qSOFA) or the National Early Warning Score 2 (NEWS2) [12] [13]. Additionally, other strategies based on the use of biomarkers such as lactate [14], C-reactive protein or procalcitonin [15], and adrenomedullin [16], the application of end-tidal carbon dioxide [17] or phenotyping [18] has been also proposed. In this sense, efforts to detect bedside sepsis by the EMS personnel are based on similar strategies to the ones adopted in the hospital setting, i.e., early warning scores, point-of-care (POC) testing, and specific training, but adapted to prehospital care [19]. However, the early identification of sepsis is still a challenge for EMS.

There is considerable interest in assessing new ways of early identification of sepsis with the $N$-terminal probrain natriuretic peptide (NT-proBNP), which is one of the most promising candidates [20]. As a result of the ongoing development of portable, robust, and reliable POC testing, it is now possible to obtain multiple biomarkers at bedside, clearly assisting the EMS personnel in the decision-making process since the very beginning of prehospital care [21].

Sepsis and septic shocks present a variable degree of multiorgan dysfunction syndromes that, in many cases, are associated to myocardial failure and with a significant rate of ICU-admissions and mortality [22, 23]. Determination of NT-proBNP is usually used to evaluate patients at risk of cardiovascular disorders, heart failure in particular, although it is also of prognostic utility in acute myocardial infarction or atrial fibrillation [24]. However, the evidence of its use in the diagnosis and stratification of sepsis is rather limited [25-28], or sparse in the prehospital scenario.

The primary endpoint of this study is aimed at determining the performance of prehospital point-of-care NTproBNP to predict sepsis, septic shock, or in-hospital sepsis-related mortality (hereafter, sepsis-related mortality includes mortality by sepsis and septic shock) and to compare its performance with qSOFA or NEWS2-gold standards in nonICU settings-scores. Secondly, we checked whether the combination of NT-proBNP with qSOFA and NEWS2 can improve their prognostic performance in suspected patients of sepsis.

\section{Methods}

2.1. Study Set-Up and Ethical Issues. The present work is a prospective, ongoing, EMS-delivered, prognostic, cohort study of adults ( $>18$ years old) evacuated by ambulance and admitted to emergency department (ED) between January 2020 and May 2021.

The study was carried out in the province of Valladolid (Spain). All calls for medical emergency help were delivered by an advanced life support (ALS) team, composed of two emergency medical technicians (EMT), an emergency registered nurse (ERN), and a physician. Once patients have been checked, cases requiring transfer were referred to the ED of the two tertiary university hospitals of the Public Health System, either in ALS or in one of the fourteen basic life support (BLS) units available in the area.

The institutional review board at the Hospital Universitario Rio Hortega and Hospital Clínico Universitario of Valladolid (reference: PI-049-19 and PI-GR-19-1258) approved the study protocol which was conducted in accordance with the Declaration of Helsinki. The study protocol was registered in the World Health Organization's International Clinical Trials Registry Platform (ICTRP) (https://doi.org/10 $.1186 /$ ISRCTN48326533), and we followed the STrengthening the Reporting of OBservational studies in Epidemiology (STROBE) [29] statement.

2.2. Population. We screened all consecutive calls for medical emergency help (1-1-2 number) received during the trial period that were dispatched by the ALS unit and finally requiring high-priority transfer to the ED (either in ALS or BLS). The study included adult patients ( $>18$ years old) with nontraumatic disease.

The following cases were excluded from the study: patients with traumatic diseases or poisoning (deliberate self-harm is classified under overdose or trauma, as appropriate), cases of cardiorespiratory arrest, pregnant women, end-of-life care situations, impossibility of conducting an analytical test through a venous blood sample, patients discharged in situ (after evaluation by the ALS physician), risky situations on the scene, and patients with no informed consent. Informed consent collection details can be found in supplementary methods.

2.3. Outcomes. The outcomes included sepsis, septic shock, and in-hospital sepsis-related mortality. For clinical operationalization, in accordance with the Third International Consensus Definitions for Sepsis and Septic Shock (Sepsis-3) [7] [30], an associate investigator reviewed the patient's electronic medical record (EMR) and collected data on sepsis (syndrome of multiorgan dysfunction with an increase of two or more SOFA score points), septic shock (deep syndrome of multiorgan dysfunction with the need for vasoactive drugs to maintain mean arterial pressure above $65 \mathrm{mmHg}$ and lactate values above $2 \mathrm{mmol} / \mathrm{L}$, after adequate fluid resuscitation), and inhospital sepsis-related mortality.

Special attention was dedicated to congestive heart failure $(\mathrm{CHF})$ as a comorbidity. A patient was considered suffering from $\mathrm{CHF}$ when the diagnosis was made by a specialist and recorded on the hospital's electronic medical record according to current heart failure guidelines [31, 32] and categorized into a subcohort for further analysis. 
All cases of sepsis, septic shock, and in-hospital sepsisrelated mortality were rechecked by the principal investigator. From now on, the terms mortality or in-hospital sepsis-related mortality will be indistinctly used.

2.4. Data Collection. All epidemiologic-demographic (age, sex, rural or urban area, intervention times, and type of ambulance) and clinical variables used to calculate qSOFA and/or NEWS2 (respiratory rate, oxygen saturation, supplemental oxygen use, systolic blood pressure, heart rate, temperature, and level of consciousness) were collected by the ERN at the scene or en route.

To determine NT-proBNP values, a trained ERN performed bedside POC by using a cobas h 232 analyzer (Roche Diagnostics, Mannheim, Germany) $[21,33,34]$ in all of the patients included in the study. The age-adjusted Charlson comorbidity index (ACCI) was used to study comorbidities $[35,36]$.

Respiratory rate, systolic blood pressure, and level of consciousness were used to determine the qSOFA [37]. In addition, oxygen saturation, heart rate, temperature, and supplemental oxygen use are included in the NEWS estimate [38]. Two or more points on the qSOFA, or 5 or more points on the NEWS and possible infection, suggest the possibility of sepsis, and additional diagnostic steps are recommended to check the suspected diagnosis $[39,40]$.

Furthers details regarding data collection can be found in supplementary methods.

2.5. Statistical Analysis. Absolute values and percentages were used for categorical variables and median and interquartile ranges (IQR) for continuous variables because they did not follow a normal distribution. The characterization of the total sample and the association between each independent variable and the outcome were assessed by the Mann-Whitney $U$ test or chi-squared test, when necessary.

Two main analyses were performed to answer the work's objectives. For the first objective, the discriminatory validity of the scores and the NT-proBNP was assessed by the area under the receiver operating characteristic (ROC) curve (AUC); specificity, sensitivity, positive predictive value, negative predictive value, positive likelihood ratio, and negative likelihood ratio of the scores and NT-proBNP were also calculated. Moreover, to compare performances, a Delong's test for AUCs comparisons and a decision curve analysis were used. To fulfill the second goal, a subset of patients was selected according to their qSOFA or NEWS scores using the following criteria: (i) patients with qSOFA scores inferior or equal to 1 and patients with scores superior or equal to 2, and (ii) for NEWS, scores superior or equal to 4 and NEWS scores superior or equal to 5. In this last case, only the AUC of the ROC was determined. The criteria for such scores' thresholds were based on the recommendations on the sepsis handling [7] and on the clinical use of NEWS2 [12].

All AUCs described in the work, except those from the second objective due to sample size, were determined from a validation cohort; i.e., two-thirds of the sample were used to fit the model and the other third to determine the validation capacity.

\section{Results}

3.1. Patient Baseline. A total of 1,360 patients with medical disease meting the inclusion criteria were enrolled in the study (see Figure 1). Patients were predominantly elderly (median age 73 years, IQR: 59-83, range 18-99) with a considerably higher ratio of males; $780(57.4 \%)$ were male and $580(42.6 \%)$ were female. The overall inpatient ratio was $67.1 \%$. The occurrence of sepsis, septic shock, and inhospital sepsis-related mortalities was $6.4 \%$ (67 cases), $4.2 \%$ (44 cases), and $6.1 \%$ (64 cases), respectively. In cases of sepsis, the ICU-admission rate was $32.4 \%$ (36 cases), standing out the use of norepinephrine (36.9\%, 41 cases) and the need for mechanical ventilation (25.2\%, 28 cases). Patient characteristics are described in Table 1.

The sepsis origin was distributed as follows: respiratory (21 cases, $31.3 \%$ ), abdominal (9 cases, 13.4\%), urinary (21 cases, $31.3 \%$ ), central nervous system (1 cases, $1.5 \%)$, skin ( 4 cases, $6 \%$ ), multifactorial ( 7 cases, 10.4\%), other (1 case, $1.5 \%$ ), and with unclear origin (3 cases, 4.5 ). The septic shock origin was distributed as follows: respiratory (14 cases, $31.8 \%$ ), abdominal (6 cases, $13.6 \%$ ), urinary (9 cases, $20.5 \%$ ), central nervous system ( 3 cases, $6.8 \%$ ), skin (4 cases, $9.1 \%$ ), multifactorial (3 cases, $6.8 \%$ ), and with unclear origin (5 cases, $11.4 \%)$.

3.2. NT-proBNP Accuracy and Comparison to Scores. The predictive validities of NT-proBNP, NEWS, and qSOFA for sepsis reached the following AUCs: 0.745 (95\% CI: $0.671-0.819$ ), 0.853 (95\% CI: 0.802-0.904), and 0.859 (95\% CI: 0.808-0.909), respectively. The same analysis was performed for septic shock reaching 0.807 (95\% CI: 0.729 0.886 ), 0.843 (95\% CI: 0.7391-0.946), and 0.822 (95\% CI: 0.681-0.963), respectively and for in-hospital sepsis-related mortality reaching 0.860 (95\% CI: 0.818-0.901), 0.845 (95\% CI: $0.772-0.916$ ), and 0.859 (95\% CI: 0.787-0.932), respectively. Further parameters of the predictive validity can be found in supplementary eTable 1. Additionally, the observed number of cases for each outcome accordingly with the scores and the NT-proBNP is shown in Figure 2, which also shows the predicted probability of each outcome according to the value of the scores or the NTproBNP levels. To determine the differences between the predictive validity of the NT-proBNP and the other two scores, Delong's test was used showing that the NTproBNP presented a statistically significant lower AUC as compared to the other two scores ( $p<0.02$ vs. NEWS; $\mathrm{p}<0.01$ vs. qSOFA) in the case of sepsis. On the contrary, no statistical differences were found in the cases of septic shock ( $p<0.622$ vs. NEWS; $p<0.878$ vs. qSOFA, both for septic shock) or mortality $(p<0.673$ vs. NEWS; $p<0.989$ vs. qSOFA, both corresponding for in-hospital sepsisrelated mortality). Supplementary Figure 2 shows the AUC comparison for each outcome and the decision curve analysis for the comparison between NT-proBNP and the other two scores, for each of the 3 outcomes. 


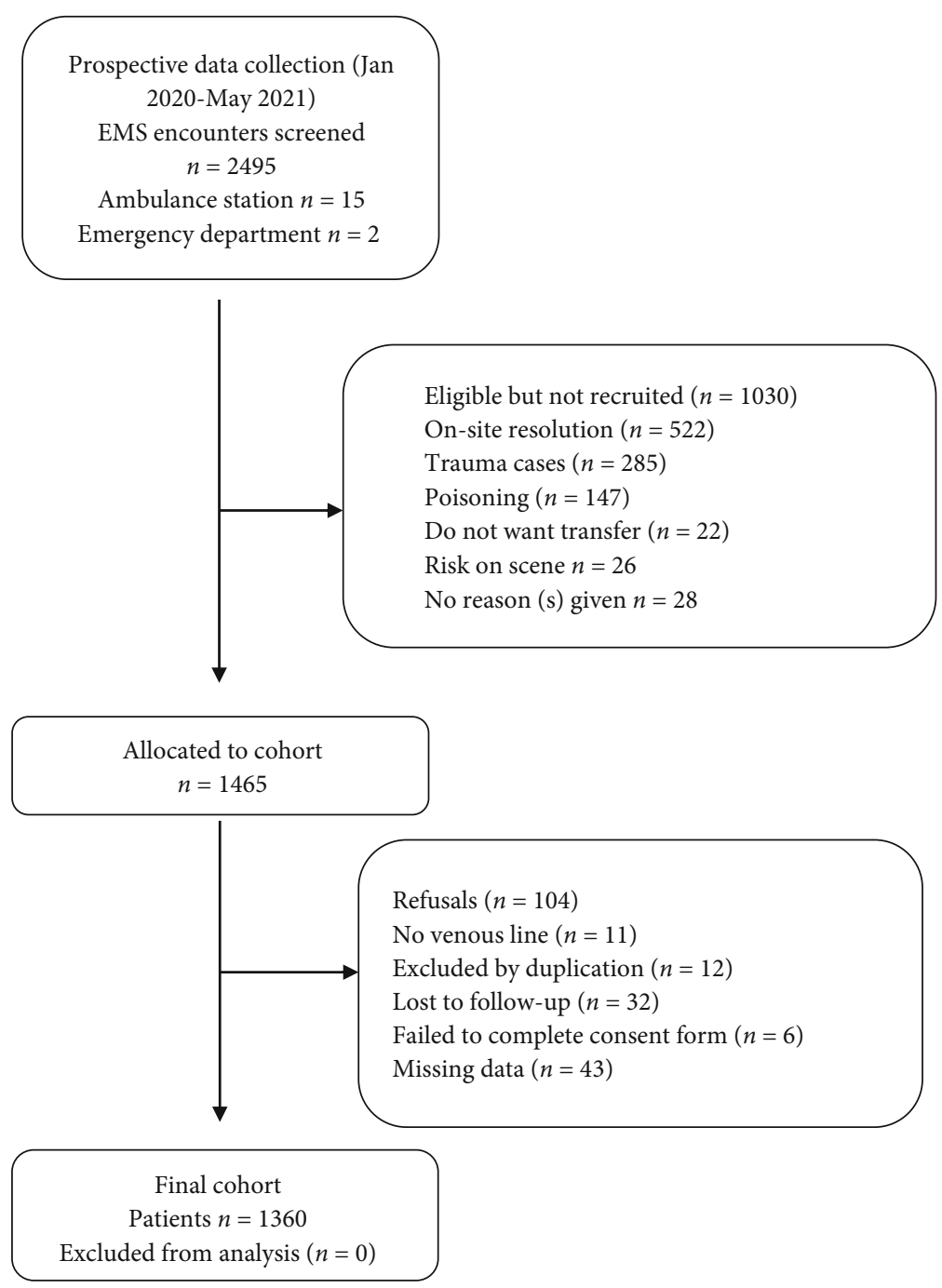

Figure 1: Participant inclusion flow diagram.

To evaluate the potential role of CHF on the scores and NT-proBNP, we compared the predictive validities of NTproBNP, NEWS, and qSOFA for the cohort of patients with and without CHF. NEWS and qSOFA did not presented statistical difference between patients with and without CHF. However, NT-proBNP presented a lower AUC for the group with $\mathrm{CHF}$ as compared to the group without CHF, but this was only statistically significant for the case of mortality (Supplementary eTable3).

3.3. NT-proBNP Added Value to NEWS and qSOFA. The second objective of this works is aimed at determining the added value of NT-proBNP on the other two scores. Only patients without CHF were used in this case. Table 2 shows the subgroup characteristics resulted from the categorization of patients according to NEWS or qSOFA. Table 3 shows the predictive validity of NT-proBNP for each subgroup. As can be observed in Table 3, the NT-proBNP inclusion in different subgroups of NEWS2 and qSOFA improved the scores' predictive validity for sepsis, septic shock, and in-hospital sepsis-related mortality. Importantly, this improvement was greater for those subgroups of low risk (NEWS $2<5$ and qSOFA $\leq 1$ ) in both scores. Further parameters of the predictive validity can be found in supplementary eTable 4.

\section{Discussion}

To our knowledge, this is the first study conducted in the prehospital care exploring the association between NTproBNP and sepsis. We found that the prehospital NEWS2 score had superior specificity and sensitivity than qSOFA and NT-proBNP for detecting sepsis and septic shock, although the NT-proBNP was better to determine patients' in-hospital sepsis-related mortality as compared to qSOFA and NEWS2.

The implementation of scoring systems in prehospital care is a clinical reality $[41,42]$. Their simplicity, the use of commonly vital signs, their elevated discriminative capacity, the easy interpretation, and their fast-learning curve make the score optimal tools for bedside use [43]. Probably the most widely implemented scoring system at an international scale is the NEWS, developed by the Royal College of 
TABLE 1: Baseline patients' characteristic-based sepsis diagnosis.

\begin{tabular}{|c|c|c|c|c|}
\hline & Total & Sepsis & Nonsepsis & $p$ value $^{\mathrm{b}}$ \\
\hline No. (\%) with data ${ }^{a}$ & 1360 & $111(8.2)$ & $1249(91.8)$ & \\
\hline Age (y) & $73(59-83)$ & $77(65-87)$ & $73(59-82)$ & .011 \\
\hline Sex (female) & $580(42.6)$ & $40(36)$ & $540(43.2)$ & .142 \\
\hline \multicolumn{5}{|l|}{ Time (minutes) } \\
\hline Arrival & $11(9-15)$ & $12(9-17)$ & $11(9-15)$ & .282 \\
\hline Assistance & $33(27-41)$ & $34(28-42)$ & $33(26-41)$ & .025 \\
\hline Transfer & $11(8-16)$ & $12(10-17)$ & $11(8-16)$ & .086 \\
\hline Advanced life support & $888(65.3)$ & $73(65.8)$ & $434(34.7)$ & .913 \\
\hline Zone (urban) & $1056(77.6)$ & $80(72.1)$ & $976(78.1)$ & .141 \\
\hline \multicolumn{5}{|l|}{ Prehospital care } \\
\hline Respiratory rate (bpm) & $18(14-26)$ & $27(23-33)$ & $18(14-24)$ & $<.001$ \\
\hline Pulse oximetry saturation (\%) & $96(92-98)$ & $92(82-95)$ & $96(93-98)$ & $<.001$ \\
\hline Supplemental oxygen & $150(11)$ & $36(32.4)$ & $114(9.1)$ & $<.001$ \\
\hline Systolic arterial pressure $(\mathrm{mmHg})$ & $137(116-158)$ & $106(88-135)$ & $139(120-160)$ & $<.001$ \\
\hline Heart rate $(\mathrm{bpm})$ & $85(70-105)$ & $104(83-124)$ & $84(70-104)$ & $<.001$ \\
\hline Temperature $\left({ }^{\circ} \mathrm{C}\right)$ & $36.1(35.8-36.7)$ & $37(36-38.6)$ & $36.1(35.8-36.6)$ & $<.001$ \\
\hline Glasgow coma scale (points) & $15(15-15)$ & $14(11-15)$ & $15(15-15)$ & $<.001$ \\
\hline Volume (mL) & $250(250-250)$ & $500(25-1000)$ & $250(250-250)$ & $<.001$ \\
\hline Mechanical ventilation & $113(8.3)$ & $17(15.3)$ & $96(7.7)$ & .005 \\
\hline Norepinephrine & $52(3.8)$ & $13(11.7)$ & $39(3.1)$ & $<.001$ \\
\hline qSOFA (points) & $1(0-1)$ & $2(1-3)$ & $0(0-1)$ & $<.001$ \\
\hline NEWS2 (points) & $4(2-8)$ & $10(7-13)$ & $4(2-7)$ & $<.001$ \\
\hline NT-proBNP (pg/mL) & $328(98-1147)$ & $1769(609-3433)$ & $300(75-1044)$ & $<.001$ \\
\hline \multicolumn{5}{|l|}{ Hospital outcomes } \\
\hline Hospital-inpatient & $912(67.1)$ & $111(100)$ & $801(64.1)$ & $<.001$ \\
\hline Hospitalization time (day) & $4(0-9)$ & $8(2-20)$ & $4(0-7)$ & $<.001$ \\
\hline Intensive care unit-admission & $139(10.2)$ & $36(32.4)$ & $103(8.2)$ & $<.001$ \\
\hline Mechanical ventilation & $131(9.6)$ & $28(25.2)$ & $103(8.2)$ & $<.001$ \\
\hline Norepinephrine & $121(8.9)$ & $41(36.9)$ & $80(6.4)$ & $<.001$ \\
\hline Septic shock & $44(3.2)$ & $44(39.6)$ & 0 & NA \\
\hline In-hospital mortality & $64(4.7)$ & $64(57.7)$ & 0 & NA \\
\hline ACCI (points) & $5(3-7)$ & $7(6-10)$ & $5(3-7)$ & $<.001$ \\
\hline AIDS & $20(1.5)$ & $2(1.9)$ & $18(1.4)$ & .762 \\
\hline Solid tumor metastatic & $65(4.8)$ & $11(9.9)$ & $54(4.3)$ & .008 \\
\hline Liver disease severe & $65(4.8)$ & $11(9.9)$ & $54(4.3)$ & .008 \\
\hline Lymphoma & $15(1.1)$ & $3(2.7)$ & $12(1)$ & .092 \\
\hline Leukemia & $18(1.3)$ & $2(1.8)$ & $16(1.3)$ & .646 \\
\hline Solid tumor localized & $314(23.1)$ & $39(35.1)$ & $275(22)$ & .002 \\
\hline DM end organ damage & $204(15)$ & $13(11.7)$ & $191(15.3)$ & .312 \\
\hline Severe chronic kidney disease & $195(14.3)$ & $25(22.5)$ & $170(13.6)$ & .010 \\
\hline Hemiplegia & $102(7.5)$ & $18(16.2)$ & $84(6.7)$ & $<.001$ \\
\hline DM uncomplicated & $204(15)$ & $13(811.7)$ & $191(15.3)$ & .312 \\
\hline Liver disease mild & $47(3.5)$ & $4(3.6)$ & $43(3.4)$ & .929 \\
\hline Peptic ulcer disease & $180(13.2)$ & $19(17.1)$ & $161(12.9)$ & .208 \\
\hline Connective disease & $125(9.2)$ & $15(13.5)$ & $110(8.8)$ & .100 \\
\hline
\end{tabular}


TABLE 1: Continued.

\begin{tabular}{|c|c|c|c|c|}
\hline & Total & Sepsis & Nonsepsis & $p$ value $^{\mathrm{b}}$ \\
\hline COPD & $303(22.3)$ & $36(32.4)$ & $267(21.4)$ & .007 \\
\hline Dementia & $210(15.4)$ & $36(32.4)$ & $174(13.9)$ & $<.001$ \\
\hline Cerebrovascular disease & $172(12.6)$ & $18(16.2)$ & $154(12.3)$ & .238 \\
\hline Peripheral vascular disease & $198(14.6)$ & $16(814.4)$ & $182(14.6)$ & .964 \\
\hline Congestive heart failure & $320(23.5)$ & $37(33.3)$ & $283(22.7)$ & .011 \\
\hline Myocardial infarction & $333(24.5)$ & $21(18.9)$ & $312(25)$ & .147 \\
\hline
\end{tabular}

qSOFA: quick Sequential Organ Failure Assessment; NEWS2: National Early Warning Score 2; NT-proBNP: $N$-terminal probrain natriuretic peptide; NA: not applicable; ACCI: age-adjusted Charlson comorbidity index; AIDS: acquired immunodeficiency syndrome; DM: diabetes mellitus; COPD: chronic obstructive

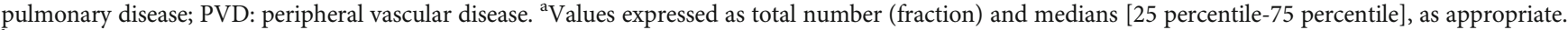
${ }^{b}$ The Mann-Whitney $U$ test, $t$-test, or chi-squared test was used as appropriate.

Physicians of London [44], with its latest update NEWS2 [12], bearing a recognized efficacy in several clinical conditions, including sepsis [38, 45]. The most widely accepted early identification score for outside-hospital of sepsis is the qSOFA, proposed in the Third International Consensus Definitions for Sepsis and Septic Shock (Sepsis-3) [7] [46].

The work presented here showed that both qSOFA and NEWS2 scores have a good forecasting capacity for the classification of sepsis, septic shock, and in-hospital sepsisrelated mortality, confirming them as fundamental tools for the diagnosis of sepsis outside the hospital settings [47, 48]. Our findings are in accordance with similar investigations, albeit with superior statistical performance. Silcock et al. [49] tested qSOFA vs. NEWS in the prehospital setting with AUCs of 0.67 and 0.74, respectively, although on unselected patients. Liu et al. [50] analyzed the predictive ability of qSOFA and NEWS in ICU-admission and in-hospital mortality in patients with suspected infection showing, an AUC of 0.78 and 0.87, respectively; Mellhammar et al. [51] obtained similar results. Nieves Ortega et al. [52] demonstrated the superior performance of NEWS over qSOFA, with an AUC of 0.85 vs. 0.79. In summary, qSOFA yielded high specificity, but low sensitivity. NEWS2 outperformed the predictive ability for sepsis-related outcomes, findings that agree with our own results $[39,53,54]$.

On the other hand, the technical possibility of bedside POC testing has encouraged EMS to implement in their guidelines the use of biomarkers in prehospital care to improve both the diagnostic and prognostic capabilities. Research concerning the clinical usefulness of prehospital NT-proBNP is scarce $[55,56]$, finding more evidence about its interpretation in patients with sepsis and especially with septic shock in the hospital setting [25, 57]. Sepsisassociated myocardial depression is a mayor expression of multiorgan dysfunction in the septic patient [58]. It is wellknown that NT-proBNP (secreted by the ventricles in response to distension or increased ischemia on demand) has a potent vasodilatory effect, inhibiting the physiological renin-angiotensin-aldosterone and the sympathetic nervous systems [26]. Recent evidence supports the suggestion that myocardial failure (and especially right ventricular dysfunction) is associated with increased short-term mortality in sepsis and septic shock [59]. About 25\% of patients with sepsis and one-half of patients with septic shock present myocardial involvement, including biventricular thickening, diminished contractility, and diastolic dysfunction [60]. Currently, the best available treatment for myocardial dysfunction in sepsis is appropriate volume-targeted resuscitation, followed by the addition of inotropes, to ensure sufficient perfusion pressure for metabolic requirements [61-63].

As a single biomarker, NT-proBNP did not improved the ability to discriminate sepsis or septic shock as compared to NEWS2 or qSOFA, nor its net benefit. Despite the fact that NT-proBNP provides encouraging performances compared to specific in-hospital biomarkers to manage sepsis, like lactate, C-reactive protein, or procalcitonin $[15,64$, 65], it did not improve bottom-line results [66]. More important, this biomarker can identify patients with sepsis and in septic shock and predict sepsis-related mortality with high reliability in the group of patients with low-risk scoring (NEWS2 $<5$ and qSOFA $\leq 1$ ) [67]. The analysis of subgroups with CHF suggested that the interpretation of NTproBNP should be handled with prudence. However, in CHF-free patients, the use of this biomarker, especially in low-risk patients (NEWS $<5$ and qSOFA $\leq 1$ ), may be helpful to identify suspected sepsis patients with an uncertain clinical course.

Patients with sepsis and septic shock have unacceptably dramatic mortality rates [68]. Despite the efforts shown by different organizations [9] [69], mortality rates remain extremely high today [70]. Sepsis is featured by fuzzy symptomatology and limited clinical manifestations in the initial stages, making its rapid detection a challenge for the EMS personnel [71]. Late recognition frequently implies that its diagnosis is often accompanied with syndromes of multiorgan dysfunction already established, delaying the therapeutic measures $[72,73]$.

\section{Limitations}

This study has several limitations. First, it is a convenience cohort, in a single province with a relatively small number of events. To minimize bias, we included 24/7 recruitments, urban and rural backgrounds, BLS or ALS 


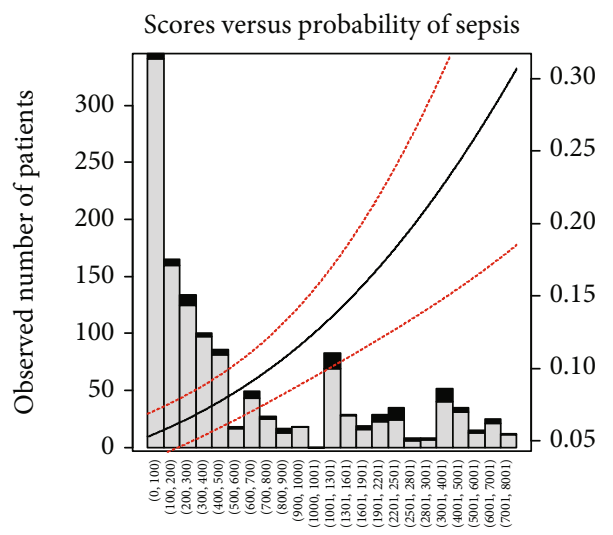

NT-proBNP levels (pg/mL)

$\square \quad$ Non-sepsis

- Sepsis

- Predicted prob.

(a)

Scores versus probability of death

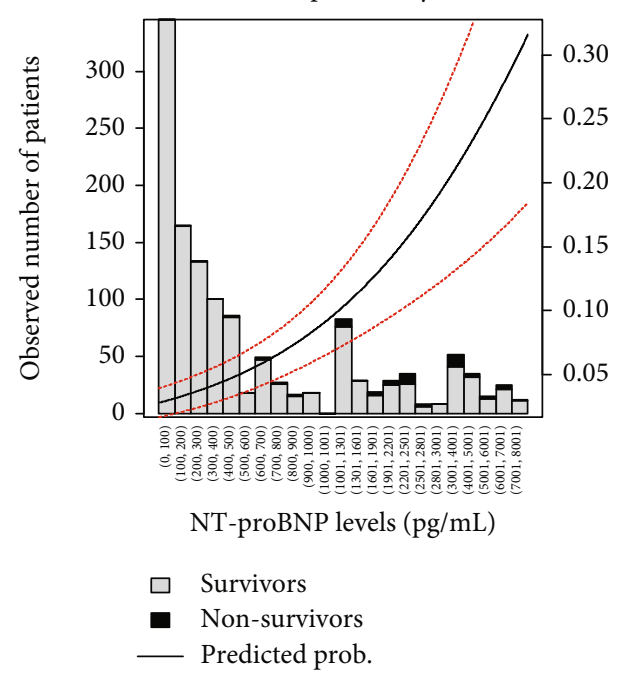

(c)

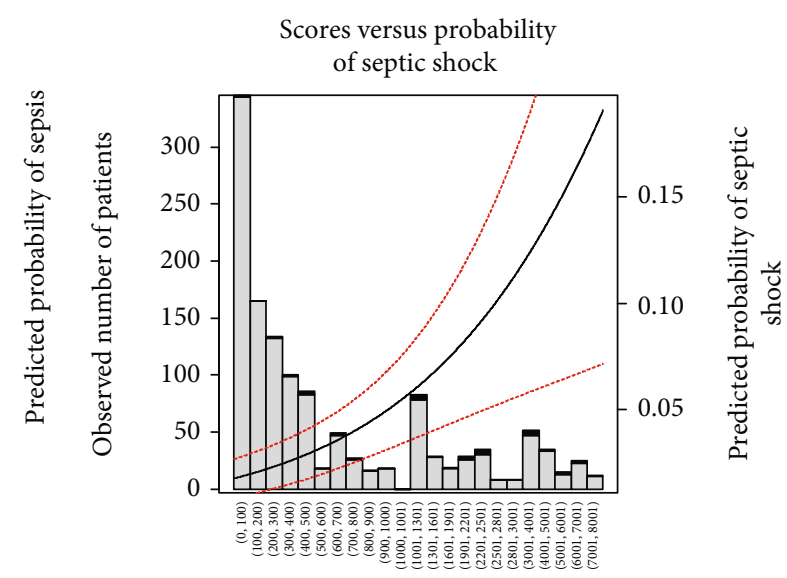

NT-proBNP levels (pg/mL)

$\square \quad$ Non-sepsis shock

- Sepsis

- Predicted prob.

(b)

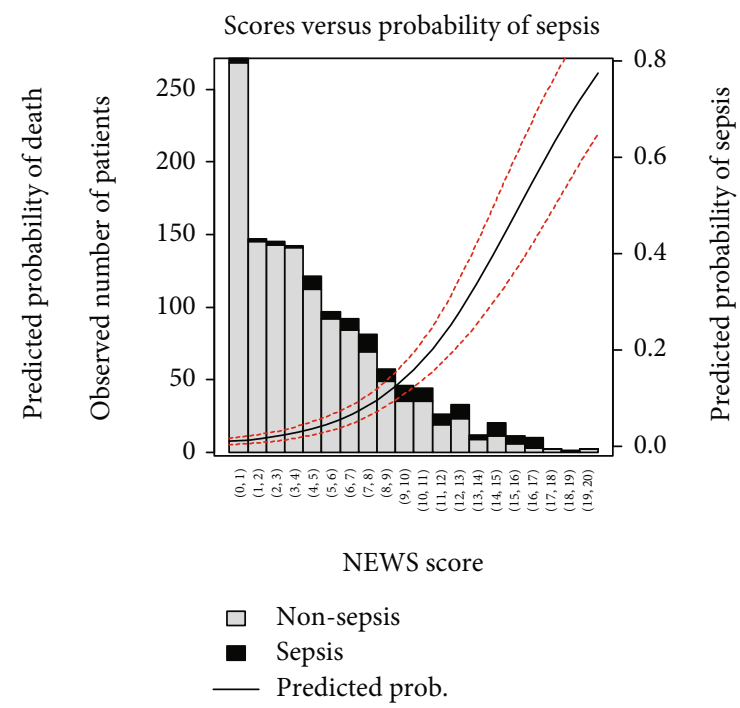

(d)

Figure 2: Continued. 


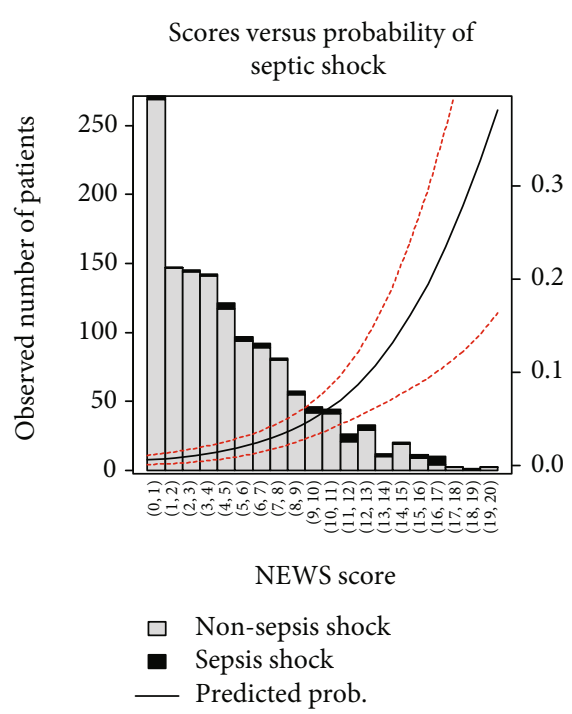

(e)

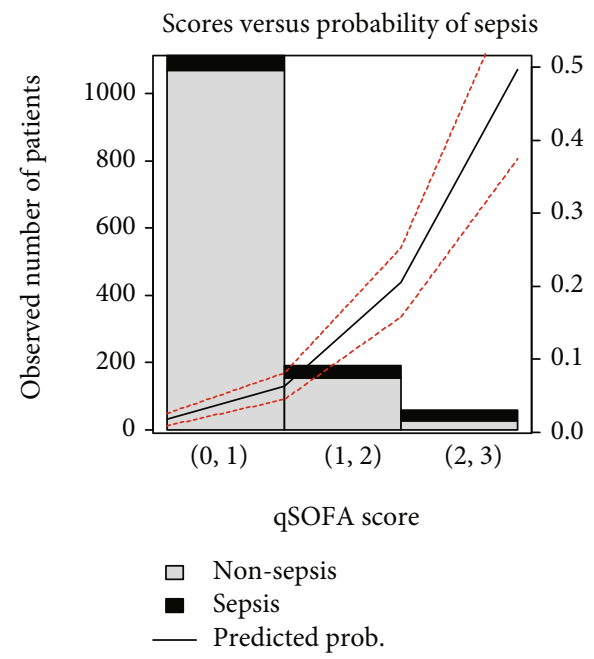

(g)

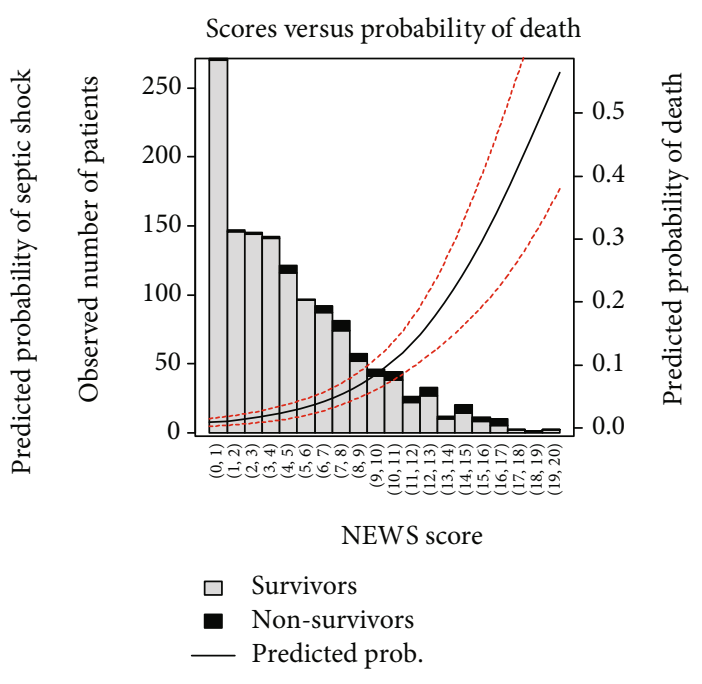

(f)

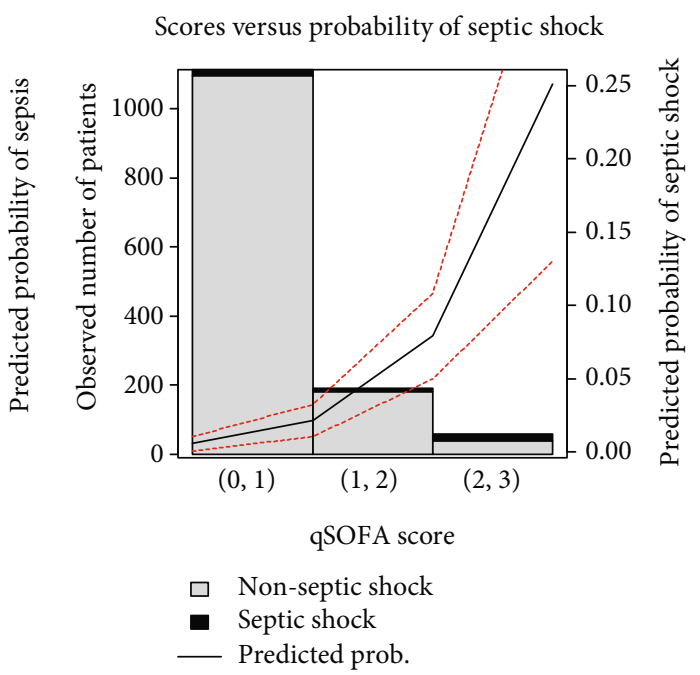

(h)

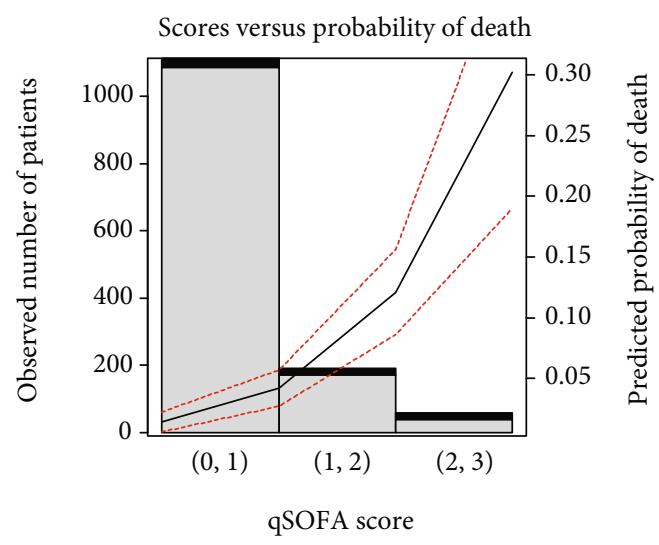

$\square \quad$ Non-sepsis

- Sepsis

- Predicted prob.

(i)

FIGURE 2: Observed number of cases for each of the outcomes: (a) sepsis, (b) septic shock, and (c) mortality for NT-proBNP; (d) sepsis, (e) septic shock, and (f) mortality for NEWS; and (g) sepsis, (h) septic shock, and (i) mortality for qSOFA. The grey shadowed area shows the predicted probability of the outcome. 
TABLE 2: Prehospital sepsis prediction using early warning scores.

\begin{tabular}{|c|c|c|c|}
\hline & Low risk & $\begin{array}{c}\text { High risk } \\
\text { Quick Sequential Organ Failure Assessment }\end{array}$ & \\
\hline & $0-1$ points & $2-3$ points & $p$ value $^{\mathrm{b}}$ \\
\hline No. (\%) with data ${ }^{\mathrm{a}}$ & $1112(81.8)$ & $248(18.2)$ & \\
\hline Age $(y)$ & $73(59-82)$ & $76(61-85)$ & .275 \\
\hline Sex (female) & $487(43.4)$ & $93(37.5)$ & .017 \\
\hline \multicolumn{4}{|l|}{ Prehospital care } \\
\hline Volume (mL) & $250(250-250)$ & $500(250-500)$ & $<.001$ \\
\hline Mechanical ventilation & $51(4.6)$ & $62(25)$ & $<.001$ \\
\hline Norepinephrine & $10(0.9)$ & $42(16.9)$ & $<.001$ \\
\hline NT-proBNP (pg/mL) & $289(67-1038)$ & $696(224-2633)$ & $<.001$ \\
\hline \multicolumn{4}{|l|}{ Hospital outcomes } \\
\hline ACCI (points) & $5(3-7)$ & $7(5-9)$ & $<.001$ \\
\hline Hospital-inpatient & $695(62.5)$ & $217(87.5)$ & $<.001$ \\
\hline Hospitalization time (day) & $4(0-8)$ & $5(1-11)$ & .008 \\
\hline Intensive care unit-admission & $83(7.5)$ & $56(22.6)$ & $<.001$ \\
\hline Mechanical ventilation & $74(6.7)$ & $57(23)$ & $<.001$ \\
\hline Norepinephrine & $53(4.8)$ & $68(27.4)$ & $<.001$ \\
\hline Sepsis & $44(4)$ & $67(27)$ & $<.001$ \\
\hline Septic shock & $16(1.4)$ & $28(11.3)$ & $<.001$ \\
\hline \multirow[t]{3}{*}{ In-hospital mortality } & $25(2.2)$ & $39(15.7)$ & $<.001$ \\
\hline & & National Early Warning Score 2 & \\
\hline & $\leq 4$ points & $\geq 5$ points & $p$ value $^{\mathrm{b}}$ \\
\hline No. (\%) with data ${ }^{a}$ & $705(51.8)$ & $655(48.2)$ & \\
\hline Age $(y)$ & $71(56-80)$ & $75(64-85)$ & $<.001$ \\
\hline Sex (female) & $283(40.1)$ & $297(45.3)$ & .053 \\
\hline \multicolumn{4}{|l|}{ Prehospital care } \\
\hline Volume (mL) & $250(250-250)$ & $250(250-500)$ & $<.001$ \\
\hline Mechanical ventilation & $9(1.3)$ & $104(15.9)$ & $<.001$ \\
\hline Norepinephrine & 0 & $52(7.9)$ & $<.001$ \\
\hline NT-proBNP (pg/mL) & $208(0-559)$ & $650(201-2399)$ & $<.001$ \\
\hline \multicolumn{4}{|l|}{ Hospital outcomes } \\
\hline ACCI (points) & $5(2-7)$ & $6(4-9)$ & $<.001$ \\
\hline Hospital-inpatient & $381(54)$ & $531(81.1)$ & $<.001$ \\
\hline Hospitalization time (day) & $3(0-7)$ & $6(1-11)$ & $<.001$ \\
\hline Intensive care unit-admission & $31(4.4)$ & $108(16.5)$ & $<.001$ \\
\hline Mechanical ventilation & $26(3.7)$ & $105(16)$ & $<.001$ \\
\hline Norepinephrine & $13(1.8)$ & $108(16.5)$ & $<.001$ \\
\hline Sepsis & $8(1.1)$ & $103(15.7)$ & $<.001$ \\
\hline Septic shock & $4(0.6)$ & $40(6.1)$ & $<.001$ \\
\hline In-hospital mortality & $4(0.6)$ & $60(9.2)$ & $<.001$ \\
\hline
\end{tabular}

NT-proBNP: $N$-terminal probrain natriuretic peptide; ACCI: age-adjusted Charlson comorbidity index; NA: not applicable. ${ }^{a}$ Values expressed as total number (fraction) and medians [ 25 percentile-75 percentile], as appropriate. ${ }^{\mathrm{b}}$ The Mann-Whitney $U$ test, $t$-test, or chi-squared test was used as appropriate.

transfers, for the duration of the follow-up; and as an add-on, validation was performed in different cohorts to check the consistency of the NT-proBNP and the scores employed. Second, the proportion of older adults is significantly elevated but does not exceed the ones of previous epidemiological studies and is in line with the general increase of elderly worldwide, especially in our neighboring countries. Third, the data extractors were not blinded. To avoid bias, the criteria of sepsis, septic shock, and in-hospital sepsis-related mortality were based on the Third International Consensus Definitions for Sepsis and Septic Shock (Sepsis-3) [7]. A physician from each hospital collected all hospital variables, and in case of sepsis-related outcomes, the event was double- 
TABle 3: Predictive validity of NT-proBNP according to NEWS and qSOFA subgroups.

\begin{tabular}{lc}
\hline Sepsis & AUC $(95 \%$ CI $)$ \\
\hline NEWS $<5$ & $0.880(0.757-1)$ \\
NEWS $\geq 5$ & $0.713(0.654-0.773)$ \\
qSOFA $\leq 1$ & $0.809(0.735-0.881)$ \\
qSOFA $>1$ & $0.705(0.621-0.788)$ \\
Septic shock & \\
NEWS $<5$ & $0.862(0.643-1)$ \\
NEWS $\geq 5$ & $0.768(0.695-0.841)$ \\
qSOFA $\leq 1$ & $0.908(0.839-0.976)$ \\
qSOFA $>1$ & $0.708(0.605-0.812)$ \\
Mortality & \\
NEWS $<5$ & $0.940(0.874-1)$ \\
NEWS $\geq 5$ & $0.828(0.772-0.885)$ \\
qSOFA $\leq 1$ & $0.903(0.855-0.950)$ \\
qSOFA $>1$ & $0.823(0.742-0.904)$ \\
\hline
\end{tabular}

AUC: area under the curve; $95 \%$ CI: $95 \%$ confidence interval. ${ }^{a}$ The low number of cases do not allow the validation procedure.

checked by the principal investigator. Fourth, although POC is clearly now being implemented in ambulances, these devices are not currently generalizable on all EMS-systems. Finally, the study began in January 2020 and stopped in May 2021, during the coronavirus 19 (COVID-19) pandemic. Broad epidemiological studies are needed to understand the impact of the ongoing pandemic on non-COVID-19 disease and identify there has been underdiagnosis of sepsis or unexpected excess mortality during this period.

\section{Conclusion}

NT-proBNP was a strong predictor of in-hospital sepsisrelated mortality, similar to the other two scores, but not for recognizing sepsis and septic shock, in which NEWS2 was better. Moreover, the NT-proBNP add-on to the other two scores improved sepsis prediction in patients at low risk of sepsis. Therefore, complementing scoring systems with POC should be a must in prehospital clinical practice since starting life support as quickly as possible is key to improve survival and minimize complications.

\section{Data Availability}

The datasets analyzed during the current study are available from the corresponding author on reasonable request.

\section{Additional Points}

Presentations. This study was not previously presented in conferences.

\section{Conflicts of Interest}

The authors declare that they have no conflicts of interest.

\section{Authors' Contributions}

Drs. Martín-Rodríguez and López-Izquierdo had full access to all of the data in the study and take responsibility for the integrity of the data and the accuracy of the data analysis. All authors are responsible for study concept and design. Delgado Benito, Álvarez Manzanares, Sanz de la Morena, Melero-Guijarro, and Teresa de la Torre de Dios are assigned to the acquisition, analysis, or interpretation of data. Martín-Rodríguez and Sanz-García drafted the manuscript. All authors did the critical revision of the manuscript for important intellectual content. Sanz García and Ortega Rabbione are responsible for the statistical analysis. Martín-Rodríguez obtained funding. Álvarez Manzanares and Sanz de la Morena are responsible for the administrative, technical, or material support. Castro Villamor worked on study supervision. Dr. Martín-Rodríguez and Melero Guijarro are joint first authors.

\section{Acknowledgments}

This work was supported by the Gerencia Regional de Salud, Public Health System of Castilla y León (Spain) [grant number GRS 1903/A/19 and GRS 2131/A/20].

\section{Supplementary Materials}

The supplementary material contains the following: Supplementary eTable 1: predictive validity of NT-proBNP according to NEWS and qSOFA subgroups. Supplementary figure 2: AUC comparison for each outcome (a) sepsis, (b) septic shock, and (c) mortality for NT-proBNP (red line), NEWS (green line), and qSOFA (blue line); and the decision curve analysis for the comparison between NT-proBNP and NEWS and qSOFA for (d) sepsis, (e) septic shock, and (f) mortality. Supplementary eTable 3: predictive validity comparison of NT-proBNP, NEWS, and qSOFA for the cohort of patients with and without CHF. Supplementary eTable 4: predictive validity of NT-proBNP according to NEWS and qSOFA. (Supplementary Materials)

\section{References}

[1] R. A. Salhi, J. M. Edwards, D. F. Gaieski, R. A. Band, B. S. Abella, and B. G. Carr, "Access to care for patients with time-sensitive conditions in Pennsylvania," Annals of Emergency Medicine, vol. 63, no. 5, pp. 572-579, 2014.

[2] M. J. Reed, A. Glover, L. Byrne et al., "Experience of implementing a national pre-hospital code red bleeding protocol in Scotland," Injury, vol. 48, no. 1, pp. 41-46, 2017.

[3] J. J. Clawson, I. Gardett, G. Scott et al., "Hospital-confirmed acute myocardial infarction: prehospital identification using the medical priority dispatch system," Prehospital and Disaster Medicine, vol. 33, no. 1, pp. 29-35, 2018.

[4] A. Kobayashi, A. Czlonkowska, G. A. Ford et al., "European Academy of Neurology and European Stroke Organization consensus statement and practical guidance for pre-hospital 
management of stroke," European Journal of Neurology, vol. 25, no. 3, pp. 425-433, 2018.

[5] M. A. Smyth, S. J. Brace-McDonnell, and G. D. Perkins, "Identification of adults with sepsis in the prehospital environment: a systematic review," BMJ Open, vol. 6, no. 8, article e011218, 2016.

[6] F. Gavelli, L. M. Castello, and G. C. Avanzi, "Management of sepsis and septic shock in the emergency department," Internal and Emergency Medicine, vol. 16, no. 6, pp. 1649-1661, 2021.

[7] M. Singer, C. S. Deutschman, C. W. Seymour et al., "The Third International Consensus Definitions for Sepsis and Septic Shock (Sepsis-3)," Journal of the American Medical Association, vol. 315, no. 8, pp. 801-810, 2016.

[8] C. W. Seymour, V. X. Liu, T. J. Iwashyna et al., "Assessment of clinical criteria for sepsis: for the Third International Consensus Definitions for Sepsis and Septic Shock (Sepsis-3)," Journal of the American Medical Association, vol. 315, no. 8, pp. 762-774, 2016.

[9] L. Evans, A. Rhodes, W. Alhazzani et al., "Surviving sepsis campaign: International Guidelines for Management of Sepsis and Septic Shock 2021," Critical Care Medicine, vol. 49, no. 11, pp. e1063-e1143, 2021.

[10] L. Evans, A. Rhodes, W. Alhazzani et al., "Executive summary: surviving sepsis campaign: International Guidelines for the Management of Sepsis and Septic Shock 2021," Critical Care Medicine, vol. 49, no. 11, pp. 1974-1982, 2021.

[11] E. P. Raith, A. A. Udy, M. Bailey et al., "Prognostic accuracy of the SOFA score, SIRS criteria, and qSOFA score for in-hospital mortality among adults with suspected infection admitted to the intensive care unit," Journal of the American Medical Association, vol. 317, no. 3, pp. 290-300, 2017.

[12] Royal College of Physicians, National Early Warning Score (NEWS) 2: Standardising the assessment of acute-illness severity in the NHS. Updated report of a working party, RCP, London, 2017.

[13] O. A. Usman, A. A. Usman, and M. A. Ward, "Comparison of SIRS, qSOFA, and NEWS for the early identification of sepsis in the emergency department," The American Journal of Emergency Medicine, vol. 37, no. 8, pp. 1490-1497, 2019.

[14] S. M. Ryoo, J. Lee, Y. S. Lee et al., "Lactate level versus lactate clearance for predicting mortality in patients with septic shock defined by sepsis-3," Critical Care Medicine, vol. 46, no. 6, pp. e489-e495, 2018.

[15] C. Pierrakos, D. Velissaris, M. Bisdorff, J. C. Marshall, and J. L. Vincent, "Biomarkers of sepsis: time for a reappraisal," Critical Care, vol. 24, no. 1, p. 287, 2020.

[16] A. Mebazaa, C. Geven, A. Hollinger et al., "Circulating adrenomedullin estimates survival and reversibility of organ failure in sepsis: the prospective observational multinational Adrenomedullin and Outcome in Sepsis and Septic Shock-1 (AdrenOSS1) study," Critical Care, vol. 22, no. 1, p. 354, 2018.

[17] C. L. Hunter, S. Silvestri, G. Ralls et al., "Comparing quick sequential organ failure assessment scores to end-tidal carbon dioxide as mortality predictors in prehospital patients with suspected sepsis," The Western Journal of Emergency Medicine, vol. 19, no. 3, pp. 446-451, 2018.

[18] C. W. Seymour, J. N. Kennedy, S. Wang et al., "Derivation, validation, and potential treatment implications of novel clinical phenotypes for sepsis," Journal of the American Medical Association, vol. 321, no. 20, pp. 2003-2017, 2019.
[19] U. M. Wallgren, J. Sjölin, H. Järnbert-Pettersson, and L. Kurland, "The predictive value of variables measurable in the ambulance and the development of the predict sepsis screening tools: a prospective cohort study," Scandinavian Journal of Trauma, Resuscitation and Emergency Medicine, vol. 28, no. 1, p. 59, 2020.

[20] H. Li, Z. Shan-Shan, K. Jian-Qiang, Y. Ling, and L. Fang, "Predictive value of C-reactive protein and NT-pro-BNP levels in sepsis patients older than 75 years: a prospective, observational study," Aging Clinical and Experimental Research, vol. 32, no. 3, pp. 389-397, 2020.

[21] N. Shimizu and K. Kotani, "Point-of-care testing of (N-terminal pro) B-type natriuretic peptide for heart disease patients in home care and ambulatory care settings," Pract Lab Med., vol. 22, article e00183, 2020.

[22] A. Arfaras-Melainis, E. Polyzogopoulou, F. Triposkiadis et al., "Heart failure and sepsis: practical recommendations for the optimal management," Heart Failure Reviews, vol. 25, no. 2, pp. 183-194, 2020.

[23] H. C. Prescott and D. C. Angus, "Enhancing recovery from sepsis: a review," Journal of the American Medical Association, vol. 319, no. 1, pp. 62-75, 2018.

[24] P. Harjola, Ò. Miró, F. J. Martín-Sánchez et al., "Pre-hospital management protocols and perceived difficulty in diagnosing acute heart failure," ESC Heart Fail., vol. 7, no. 1, pp. 289-296, 2020.

[25] S. Vallabhajosyula, Z. Wang, M. H. Murad et al., "Natriuretic peptides to predict short-term mortality in patients with sepsis: a systematic review and meta-analysis," Mayo Clin Proc Innov Qual Outcomes., vol. 4, no. 1, pp. 50-64, 2020.

[26] X. Zhou, S. Wu, and Y. Ye, "Accuracy and reliability of brain natriuretic peptide (BNP) in predicting the prognosis of noncardiac patients with sepsis," Journal of Critical Care, vol. 44, pp. 475-476, 2018.

[27] B. Bhandari and J. Cunningham, "The role of brain natriuretic peptide as a prognostic marker for sepsis," Cureus., vol. 12, no. 7, article e8954, 2020.

[28] J. Khoury, M. Arow, A. Elias et al., "The prognostic value of brain natriuretic peptide (BNP) in non-cardiac patients with sepsis, ultra-long follow-up," Journal of Critical Care, vol. 42, pp. 117-122, 2017.

[29] E. von Elm, D. G. Altman, M. Egger, S. J. Pocock, P. C. Gøtzsche, and J. P. Vandenbroucke, "The Strengthening the Reporting of Observational Studies in Epidemiology (STROBE) statement: guidelines for reporting observational studies,” Lancet, vol. 370, no. 9596, pp. 1453-1457, 2007.

[30] M. Shankar-Hari, G. S. Phillips, M. L. Levy et al., "Developing a new definition and assessing new clinical criteria for septic shock: for the Third International Consensus Definitions for Sepsis and Septic Shock (Sepsis-3)," Journal of the American Medical Association, vol. 315, no. 8, pp. 775-787, 2016.

[31] P. van der Meer, H. K. Gaggin, and G. W. Dec, "ACC/AHA versus ESC Guidelines on heart failure:," Journal of the American College of Cardiology, vol. 73, no. 21, pp. 2756-2768, 2019.

[32] C. Mueller, K. McDonald, R. A. de Boer et al., "Heart Failure Association of the European Society of Cardiology practical guidance on the use of natriuretic peptide concentrations," European Journal of Heart Failure, vol. 21, no. 6, pp. 715731, 2019.

[33] A. M. R. Schols, J. P. G. Stakenborg, G. J. Dinant, R. T. A. Willemsen, and J. W. L. Cals, "Point-of-care testing in primary care 
patients with acute cardiopulmonary symptoms: a systematic review," Family Practice, vol. 35, no. 1, pp. 4-12, 2018.

[34] Y. Pan, D. Li, J. Ma, L. Shan, and M. Wei, "NT-proBNP test with improved accuracy for the diagnosis of chronic heart failure," Medicine (Baltimore), vol. 96, no. 51, article e9181, 2017.

[35] N. W. Setter, M. L. Peres, B. M. M. de Almeida, R. R. Petterle, and S. M. Raboni, "Charlson comorbidity index scores and inhospital prognosis of patients with severe acute respiratory infections," Internal Medicine Journal, vol. 50, no. 6, pp. 691-697, 2020.

[36] M. Saji, M. R. Katz, G. Ailawadi, D. E. Fowler, M. Ragosta, and D. S. Lim, "Predictive value of age-adjusted Charlson comorbidity index for 1-, 3-, and 5-year mortality in patients requiring transcatheter mitral valve repair," The American Journal of Cardiology, vol. 120, no. 2, pp. 309-314, 2017.

[37] T. Hirose, Y. Katayama, H. Ogura et al., "Relationship between the prehospital quick Sequential Organ Failure Assessment and prognosis in patients with sepsis or suspected sepsis: a population-based ORION registry," Acute Med Surg., vol. 8, no. 1, article e675, 2021.

[38] M. Maciver, "Pre-hospital use of early warning scores to improve detection and outcomes of sepsis," British Journal of Community Nursing, vol. 26, no. 3, pp. 122-129, 2021.

[39] A. F. Oduncu, G. S. Kıyan, and S. Yalçınlı, "Comparison of qSOFA, SIRS, and NEWS scoring systems for diagnosis, mortality, and morbidity of sepsis in emergency department," The American Journal of Emergency Medicine, vol. 48, pp. 54-59, 2021.

[40] G. H. Latten, J. Polak, A. H. Merry et al., "Frequency of alterations in qSOFA, SIRS, MEWS and NEWS scores during the emergency department stay in infectious patients: a prospective study," International Journal of Emergency Medicine, vol. 14, no. 1, p. 69, 2021.

[41] T. A. Williams, H. Tohira, J. Finn, G. D. Perkins, and K. M. Ho, "The ability of early warning scores (EWS) to detect critical illness in the prehospital setting: a systematic review," Resuscitation, vol. 102, pp. 35-43, 2016.

[42] R. Patel, M. D. Nugawela, H. B. Edwards et al., "Can early warning scores identify deteriorating patients in pre-hospital settings? A systematic review A systematic review," Resuscitation, vol. 132, pp. 101-111, 2018.

[43] F. Martín-Rodríguez, A. Sanz-García, E. Medina-Lozano et al., "The value of prehospital early warning scores to predict in hospital clinical deterioration: a multicenter, observational base-ambulance study," Prehospital Emergency Care, vol. 25, no. 5, pp. 597-606, 2021.

[44] Royal College of Physicians, National Early Warning Score (NEWS): Standardising the assessment of acuteillness severity in the NHS. Report of a working party, RCP, London, 2012.

[45] A. Pullyblank, A. Tavaré, H. Little et al., "Implementation of the National Early Warning Score in patients with suspicion of sepsis: evaluation of a system-wide quality improvement project," The British Journal of General Practice, vol. 70, no. 695, pp. e381-e388, 2020.

[46] E. Shu, C. Ives Tallman, W. Frye et al., "Pre-hospital qSOFA as a predictor of sepsis and mortality," The American Journal of Emergency Medicine, vol. 37, no. 7, pp. 1273-1278, 2019.

[47] M. M. Churpek, A. Snyder, X. Han et al., "Quick sepsis-related organ failure assessment, systemic inflammatory response syndrome, and early warning scores for detecting clinical deterioration in infected patients outside the intensive care unit,"
American Journal of Respiratory and Critical Care Medicine, vol. 195, no. 7, pp. 906-911, 2017.

[48] D. J. Lane, H. Wunsch, R. Saskin et al., "Screening strategies to identify sepsis in the prehospital setting: a validation study," CMAJ, vol. 192, no. 10, pp. E230-E239, 2020.

[49] D. J. Silcock, A. R. Corfield, H. Staines, and K. D. Rooney, "Superior performance of National Early Warning Score compared with quick Sepsis-related Organ Failure Assessment Score in predicting adverse outcomes: a retrospective observational study of patients in the prehospital setting," European Journal of Emergency Medicine, vol. 26, no. 6, pp. 433-439, 2019.

[50] V. X. Liu, Y. Lu, K. A. Carey et al., "Comparison of early warning scoring systems for hospitalized patients with and without Infection at risk for in-hospital mortality and transfer to the intensive care unit," JAMA Network Open, vol. 3, no. 5, article e205191, 2020.

[51] L. Mellhammar, A. Linder, J. Tverring et al., "NEWS2 is superior to qSOFA in detecting sepsis with organ dysfunction in the emergency department," Journal of Clinical Medicine, vol. 8, no. 8, p. 1128, 2019.

[52] R. Nieves Ortega, C. Rosin, R. Bingisser, and C. H. Nickel, "Clinical scores and formal triage for screening of sepsis and adverse outcomes on arrival in an emergency department allcomer cohort," The Journal of Emergency Medicine, vol. 57, no. 4, pp. 453-460.e2, 2019.

[53] R. Goulden, M. C. Hoyle, J. Monis et al., "qSOFA, SIRS and NEWS for predicting inhospital mortality and ICU admission in emergency admissions treated as sepsis," Emergency Medicine Journal, vol. 35, no. 6, pp. 345-349, 2018.

[54] M. Dorsett, M. Kroll, C. S. Smith, P. Asaro, S. Y. Liang, and H. P. Moy, "qSOFA has poor sensitivity for prehospital identification of severe sepsis and septic shock," Prehospital Emergency Care, vol. 21, no. 4, pp. 489-497, 2017.

[55] M. T. Bøtker, M. T. Jørgensen, C. Stengaard et al., "Prehospital triage of patients suffering severe dyspnoea using N-terminal pro-brain natriuretic peptide, the PreBNP trial: a randomised controlled clinical trial," European Heart Journal Acute Cardiovascular Care, vol. 7, no. 4, pp. 302-310, 2018.

[56] D. Harpaz, R. C. S. Seet, R. S. Marks, and A. I. Y. Tok, "B-type natriuretic peptide as a significant brain biomarker for stroke triaging using a bedside point-of-care monitoring biosensor," Biosensors (Basel)., vol. 10, no. 9, p. 107, 2020.

[57] L. Kakoullis, E. Giannopoulou, E. Papachristodoulou et al., "The utility of brain natriuretic peptides in septic shock as markers for mortality and cardiac dysfunction: a systematic review," International Journal of Clinical Practice, vol. 73, no. 7, article e13374, 2019.

[58] H. S. Jeong, T. H. Lee, C. H. Bang, J. H. Kim, and S. J. Hong, "Risk factors and outcomes of sepsis-induced myocardial dysfunction and stress-induced cardiomyopathy in sepsis or septic shock: a comparative retrospective study," Medicine (Baltimore), vol. 97, no. 13, article e0263, 2018.

[59] S. Vallabhajosyula, A. Shankar, R. Vojjini et al., "Impact of right ventricular dysfunction on short-term and long-term mortality in sepsis: a meta-analysis of 1,373 patients," Chest, vol. 159, no. 6, pp. 2254-2263, 2021.

[60] T. W. Jones, S. E. Smith, J. S. Van Tuyl, and A. S. Newsome, "Sepsis with preexisting heart failure: management of confounding clinical features," Journal of Intensive Care Medicine, vol. 36, no. 9, pp. 989-1012, 2021. 
[61] R. J. Gazmuri and C. A. de Gomez, "From a pressure-guided to a perfusion-centered resuscitation strategy in septic shock: critical literature review and illustrative case," Journal of Critical Care, vol. 56, pp. 294-304, 2020.

[62] N. Simpson, F. Lamontagne, and M. Shankar-Hari, "Septic shock resuscitation in the first hour," Current Opinion in Critical Care, vol. 23, no. 6, pp. 561-566, 2017.

[63] T. W. L. Scheeren, J. Bakker, T. Kaufmann et al., "Current use of inotropes in circulatory shock," Annals of Intensive Care, vol. 11, no. 1, p. 21, 2021.

[64] P. E. Marik and E. Stephenson, "The ability of procalcitonin, lactate, white blood cell count and neutrophil- lymphocyte count ratio to predict blood stream infection. Analysis of a large database," Journal of Critical Care, vol. 60, pp. 135-139, 2020.

[65] C. T. Lin, J. J. Lu, Y. C. Chen, V. C. Kok, and J. T. Horng, "Diagnostic value of serum procalcitonin, lactate, and highsensitivity C-reactive protein for predicting bacteremia in adult patients in the emergency department," PeerJ, vol. 5, article e4094, 2017.

[66] S. Spoto, E. Nobile, E. P. Carnà et al., "Best diagnostic accuracy of sepsis combining SIRS criteria or qSOFA score with procalcitonin and mid-regional pro-adrenomedullin outside ICU," Scientific Reports, vol. 10, no. 1, p. 16605, 2020.

[67] R. Liu, N. S. Chaudhary, D. M. Yealy, D. T. Huang, and H. E. Wang, "Emergency medical services care and sepsis trajectories," Prehospital Emergency Care, vol. 24, no. 6, pp. 733-740, 2020.

[68] C. Fleischmann-Struzek, L. Mellhammar, N. Rose et al., "Incidence and mortality of hospital- and ICU-treated sepsis: results from an updated and expanded systematic review and meta-analysis," Intensive Care Medicine, vol. 46, no. 8, pp. 1552-1562, 2020.

[69] M. M. Levy, L. E. Evans, and A. Rhodes, "The surviving sepsis campaign bundle," Critical Care Medicine, vol. 46, no. 6, pp. 997-1000, 2018.

[70] K. E. Rudd, S. C. Johnson, K. M. Agesa et al., "Global, regional, and national sepsis incidence and mortality, 1990-2017: analysis for the Global Burden of Disease Study," Lancet, vol. 395, no. 10219, pp. 200-211, 2020.

[71] C. Metelmann, B. Metelmann, C. Scheer et al., "Sepsis detection in emergency medicine : results of an interprofessional survey on sepsis detection in prehospital emergency medicine and emergency departments," Der Anaesthesist, vol. 67, no. 8, pp. 584-591, 2018.

[72] L. Gripp, M. Raffoul, and K. A. Milner, "Implementation of the Surviving Sepsis Campaign one-hour bundle in a short stay unit: a quality improvement project," Intensive \& Critical Care Nursing, vol. 63, article ???, 2021.

[73] O. Sjösten, J. Nilsson, J. Herlitz, C. Axelsson, M. Jiménez-Herrera, and H. M. Andersson, "The prehospital assessment of patients with a final hospital diagnosis of sepsis: results of an observational study," Australas Emerg Care, vol. 22, no. 3, pp. 187-192, 2019. 